RUNNING HEAD: Computational modeling of person-situation transactions

Computational modeling of person-situation transactions: How accumulation of situational experiences can shape the distributions of trait scores

\author{
René Mõttus \\ Mike Allerhand \\ Wendy Johnson \\ Department of Psychology, Centre for Cognitive Ageing and Cognitive Epidemiology \\ University of Edinburgh
}

rene.mottus@ed.ac.uk

7 George Square

EH8 9JZ Edinburgh

Scotland, UK 


\begin{abstract}
The chapter argues that individuals are dynamic systems that continually interact with their situational experiences. If so, then in addition to linking particular situational experiences with particular personality characteristics, research could address general principles of personsituation interactions. It is argued that computational modeling may be provide a useful set of tools for generating and refining hypotheses regarding such general principles. It encourages conceptual rigor as it requires carefully thought-through and mathematically formulated hypotheses and it also allows proof-of-principle testing of the hypotheses, although it certainly does not provide empirical evidence. Specifically, this chapter is concerned with two kinds of situational influences on the magnitude of individual differences. First, based on previous theorizing, it is hypothesizes that person-environment transactions whereby situational experiences reflect and reinforce pre-existing personality characteristics increase the magnitude of individual differences. For comparison, existent empirical findings point increases of personality variance in childhood and no systematic developmental trends thereafter, which has been problematic for the hypothesis that individuals systematically transact with their environments. Second, it is hypothesized that random influences may decrease the magnitude of individual differences, potentially counteracting the alleged variance-increasing tendency of systematic transactions. The chapter introduces a framework (personality space framework; PSF) for building computational models that allow playing through such scenarios. Simulations based on the computational model pointed to the possibility that any kind of accumulating situational experiences may tend to make people more alike until variance reaches a plateau, regardless of whether the experiences are random or systematically tied to pre-existing trait levels.
\end{abstract}




\section{Computational modeling of person-situation transactions: How accumulation of situational experiences can shape the distributions of trait scores}

In this chapter, we are interested in how accumulating situational experiences may potentially influence psychological development. Specifically, we focus on one observable aspect of development: the magnitudes of individual differences in personality characteristics. We hypothesize that relatively specific external circumstances — situations as people experience them -accumulate over time and can continually influence, or update, individuals' pre-existing personality characteristics. Therefore, we do not define external influences as broad classes of influences that aggregate multitudes of specific factors or situational experiences (e.g., genes, parental family environment, having a family of one's own, living in a Western culture) or reflect major but rare life events (e.g., trauma, child-birth, losing job). Instead, we consider the possible role of relatively specific and mundane situations that may each last for only short periods of time and be confined to particular physical or social circumstances.

We are interested in how the process of the the accumulation of such situational experiences can play out with regard to variability in personality characteristics. Perhaps unlike many other chapters in the book, we will not label the kinds of situations that we consider or characterize them in detail other than treating them as falling into one of two broad types: random situational experiences or those reflecting systematic person-social environment transactions. The defining feature of the latter class is that the situational experiences are partly dependent on people's pre-existing characteristics. Of course, there are various other classes of situational experiences that people can face, but here we focus on only these two. 


\section{Computational modeling as a potentially useful tool}

We propose that one way to systematically and rigorously think about complex psychological phenomena such as accumulation of situational experiences and their interactions with personality characteristics is to formulate working hypotheses as computational models. Essentially, this means that hypotheses are set up in mathematical terms and thereby lend themselves to being implemented as computer programs that take some specified input values and, on the basis of these input values and clearly specified algorithms that operationalize specific hypotheses, produce some numerical outputs. We emphasize that these numerical outputs do not provide empirical evidence for the hypotheses that they purportedly reflect because they entirely depend on the input values and algorithms going in. (To some extent, statistical analyses of real empirical data are subject to the researcher-imposed algorithmic choices. For example, correlation coefficients reflect our preconceived idea that the phenomena of interest have linear associations across the full range of their values. As we will argue below, this may not plausible at least in some scenarios, but the assumption nevertheless influences the outcome of the analyses.)

Instead, computational models may be useful thinking tools. First, they encourage-in fact, even require - clarity and rigor in thinking. For an idea to be mathematically specified and thereby implementable as a computational model, it has to be fleshed out in considerable detail. For example, nothing is easier than saying that people choose much about the situations they experience and the situations they experience in turn influence them. To make this idea specific enough to be implemented as a computational model requires operationalizing individuals, situations and the specific manner in which they transact. Indeed, getting a model that involves a 
number of interacting parameters to run at all may require a great deal of hard thinking. If there are blatant gaps or inconsistencies in how the ideas are operationalized, the model simply will not run or will produce values that are clearly outside any plausible boundaries for them. Of course, a running model generating reasonable-looking outputs does not inevitably tell us something useful about the real world. But it may. And perhaps more importantly, a model that does not even run has less even chance of being useful.

Second, to the extent that the output of one computational model bears more resemblance to the phenomena that we observe in the real world than the output of some other models, the hypotheses on which this model was based would appear somewhat more plausible and perhaps, just perhaps more worthwhile of being submitted to empirical tests. Collecting real data is often expensive, so proof-of-principle testing of ideas before submitting them to empirical tests may make a lot of sense. Finally, when ideas fail as computational models, they can be "tweaked" until they start working. This may lead to new hypotheses, as may models' side-products: aspects of models' behavior that were not intended in the first place, but may be interesting nevertheless. In sum, thus, computational models are tools that can guide thinking prior to empirical testing.

Of course, computational modeling is already being employed in psychological science (e.g., Gershman, Horvitz, \& Tenenbaum, 2015), but it has not been extensively used in personality research (cf. Fraley \& Roberts, 2005; Read et al., 2010), never mind in attempts to study the transactions between personality characteristics and situations. We therefore describe a novel framework for building computational models that could be suitable for playing through complex scenarios that involve numerous variables and their transactions - such as those between situations and personality characteristics. 


\section{Personality variance}

In one sense, variance has always played a central role in personality research, to the extent that personality is often defined as variance- that is, individual differences. Pivotal conceptual-mathematical tools in personality research such as principal component and factor analyses or correlation are based on variance, as are attempts to link personality characteristics to their possible causes (e.g., Bjørnebekk et al., 2013) and consequences (e.g., Roberts, Kuncel, Shiner, Caspi, \& Goldberg, 2007). Little variance among individuals in some behavior literally means little involvement of personality. Recently, another level of personality variance - that expressed within people over time and across situations - has become a popular subject of study, with studies consistently showing that there may be even more variance within individuals than among them (Mõttus, Epskamp, \& Francis, in press; Sherman, Rauthmann, Brown, Serfass, \& Jones, 2015). In another and more explicit sense, however, variance in personality characteristics has been paid surprisingly little explicit attention. For example, although a multitude of research has investigated how average levels of personality characteristics are associated with, say, life experiences or demographic features such as age and gender or cultural/national groupings, research into how these features are linked with variance in personality characteristics is rare.

We think that this ought to change, because variance patterns may provide useful insights. Variance may tell us something how and which influences operate on personality. One field of research that may benefit from attention to variance is personality development. For example, it has been proposed that individuals' personalities develop by transacting with their environments (Caspi \& Roberts, 2001; Caspi, Roberts, \& Shiner, 2005), with people tending to select themselves into, or create for themselves, environmental experiences that match their pre-existing 
personality characteristics and these self-selected experiences then reinforcing the characteristics. In the context of this handbook, we can think of environments as recurring experiences of systematically similar situations.

Indeed, it has been suggested for quite some time that the life experiences that people actively seek, create or evoke may deepen the personality characteristics that led to these experiences in the first place, a phenomenon referred to as the corresponsive principle of personality development (Caspi et al., 2005). For example, extraverted people may systematically seek out socially stimulating situations, or perceive situations as suitable for social engagements or modify them to be such, and the accumulation of the resulting experiences might further enhance their social skills and thereby gradually accentuate their extraversion even more. In contrast, less extraverted individuals may avoid socially stimulating situations and thereby deprive themselves of practicing relevant skills, which may further lessen their social ambitions and thereby decrease their level of extraversion even more. If and when the corresponsive principle holds, it would suggest that individual differences in personality characteristics ought to increase over time. As people advance in age, they are likely to have accumulated opportunities to select themselves into matching situations and receive reinforcing influences from the resulting experiences. Therefore, ceteris paribus, individuals who initially score relatively high on a trait should tend to score yet higher, individuals who initially score relatively low on the trait should become lower still, and overall variance on the trait should increase over time.

This (verbal) reasoning seems right within the context of considering extraversion, or any single trait, in isolation from everything else, but no trait likely develops independently of all other traits and life circumstances that influence these. In each individual, there is probably a 
complicated system of multiple characteristics that transact with each other (Cramer et al., 2012; Mõttus \& Allerhand, in press)—-some contributing to or inhibiting one or more others — and also with multiple situational experiences over numerous occasions. This means thousands or even millions of specific processes when we consider these processes as happening continually over time. This is something that is very hard to build into any verbal model with any tractable level of specificity. It may be that the expected tendency for increasing variance just never becomes observable because of the sheer number of transactions in individuals' personality systems. Or, alternatively, it may be that the tendency, at least as per the one-trait-at-a-time hypothesis, in fact plays out so strongly over the course of these numerous transactions as to lead to unrealistically large increases in variance - an outcome that should also question the idea of person-environment transactions increasing variance. It is difficult to mentally play through these scenarios to assess their plausibility.

Mõttus and colleagues (2016) suggested that the plausibility of the hypothesis of personsituation transactions increasing variance could be tested using a simulation design, which means implementing it as a computational model. This could have at least two benefits. First, it could make us think rigorously about some of the specif implications of the hypothesis, because this is necessary to formulate the hypothesis in mathematical terms. Second, to the extent that the computational model produces results that are consistent with the hypothesis, it could lend some proof-of-principle credibility to it and thereby make a stronger case for attempts to test it empirically. Or the results could suggest modifying the hypothesis, or perhaps scrapping it altogether.

Naturally, if some empirical findings already exist, the computational results can be 
compared to these. In empirical data, variance in personality characteristics does not appear to systematically change from adolescence onwards (Mõttus et al., 2016). However, there is a robust trend for increasing variance from childhood through early adolescence, although the effect sizes are not large (their exact magnitude is hard to quantify because it depends on measurement scales; Mõttus, Soto, \& Slobodskaya, in revision). Given these findings, if it appears plausible that the corresponsive principle indeed entails increases in variance, ceteris paribus. But because the tendency is not what is empirically observed in adulthood, this would suggest that the either the corresponsive principle no longer applies or there are some countervailing forces that offset its implications. In the latter case, the hypothesis needs to be tweaked accordingly.

Indeed, there may be other influences that negate or even reverse the possible varianceincreasing tendencies of person-situation transactions (Mõttus et al., 2016). For instance, the maturity principle of personality development postulates that the typical mean-level changes that occur during adulthood-with most people becoming more socially dominant, agreeable, conscientious, and emotionally stable with age-reflect socialization processes that act to moderate or negate tendencies to express all traits in extremity due to negative social consequences (Caspi et al., 2005). Such socialization processes would tend to make individuals more alike-more similar to the "average" or most socially-well-adapted person-thereby reducing trait variance.

Moreover, personality development may be subject to essentially random situational influences that are neither related to pre-existing trait levels (as per person-environment transactions) nor tend to pull everyone toward particular trait levels (through socialization processes). For example, having a friendly desk-mate at office or being bumped into in the 
subway might be considered random influences that could but do not have to happen to anyone, regardless of their initial personality characteristics. Of course, even such influences may be more likely to happen to people with some characteristics, but let's consider the possibility that even within the situational experiences that people work towards creating for themselves and that match their characteristics some aspects of the situations are uncontrollable.

To the extent that such random situational influences exist, their accumulation over time could tend to pull individuals toward average personality profiles rather than promote extreme trait levels. This is because situations that are average in terms of their personality-relevant aspects may be more likely than those contributing toward the extreme trait levels - traits are normally distributed and so can be people's everyday experiences, conceptualized along some psychologically relevant dimensions. Among other factors, this may be because many situations are social in nature and thereby reflect the traits of other people-and these are normally distributed. But this may also happen for purely mathematical reasons: according to the central limit theorem, average occurrences of a large number of independent variables (e.g., different types of experiences that are relevant for a particular trait but that are independent by virtue of being random) tend to form a normal-like distribution, regardless of their own distributions.

Thus, while the influences of possible socialization pressures on variance are self-evident - people tend to respond to common social pressures by adopting similar habitual adaptive behavior patterns, which thereby restricts variance-the hypothesis pertaining to random situational influences is more complex and its implications for variance may be arguable: we may not be able to anticipate likely patterns by mere verbal reasoning. Therefore, we may gain insights about possible implications of random influences by using computational models. 
There may be other reasons that variance in personality characteristics increases in childhood and not after this. One is that a trend toward increasing variance due to transactions between personality characteristics and situational experiences may not be linear. Specifically, characteristics might become inured to accumulating situational experiences, because they may not be infinitely malleable. Once some characteristics have been pulled from their baseline levels by, say, systematic situational experiences, it may become ever more difficult to pull them yet further in the particular direction. Among other reasons, this may happen as the responses to these situations become habitual or because trait-expression has some physical/biological limits (that may also vary across people, of course) - one can be only be talkative or argumentative to a certain degree. If one thinks of the baseline levels as genetically influenced, then this hypothesis reflects a non-linear form of gene-environment interaction. Genetic influences predispose individuals to particular situational experiences that initially foster the manifestation of these influences, but then the characteristics being influenced become increasingly insensitive to, or saturated by, these influences and the fostering effect of the environment wanes.

In the next two sections, we describe a computational framework that we call personality space framework (PSF). The framework is suitable for modeling hypotheses that relate accumulation of situational experiences to the development of the distributions of personality characteristics. It represents individuals as dynamic systems that "consist of" multiple interacting characteristics, form groups of transacting systems (i.e., individuals who coalesce into groups), and adhere to principles of self-organization. The possibility that individuals transact based on their characteristics is particularly important, because this allows for a form of personenvironment transactions that can be modeled. Here we consider the possibility that individuals 
tend to transact with and be influenced by others who are similar to them in personality characteristics. In real life this may happen because individuals' choices of situations are based on their pre-existing characteristics (Bahns, Crandall, Gillath, \& Preacher, 2016) or because they directly prefer like-minded others (Grosz, Dufner, Back, \& Denissen, 2015; Selfhout et al., 2010). Of course, other principles of inter-personal dynamics could also be modeled. Therefore, what we mean by situations is perceptions of other individuals.

\section{Conceptual description of the PSF}

Common techniques in personality research such as principal component analysis (PCA) represent individuals as points or vectors in a multidimensional feature space. A central concept of the PSF is personality space, which is essentially a dynamic version of a multidimensional feature space, or a model of the manner that can accommodate individuals, their interactions and development over time. Assuming that personality features can be represented by dimensional quantities, we can project individuals into a multidimensional space spanned by orthogonal dimensions representing the individual features considered in the model. Each individual can then be thought of as a point in this feature space, the coordinates of which are the quantities of the individual on the features. In other words, individuals' co-ordinates in personality space represent their personality profiles, as sometimes studied in what is called the person-centered approach (Asendorpf, 2015). In line with much of personality research, we call these features traits, but it is important to note that here (as in much of personality research) they are essentially just placeholders. For example, traits may represent very specific behavioral tendencies in addition to, or even instead of, the broad aggregate dispositions the term often denotes in personality literature. Although some traits may be unique to single individuals or appear and disappear as individuals 
develop, here we assume that there exists a set of traits on which all individual are scalable throughout observable development ${ }^{1}$. Equally, individuals can be thought of as vectors starting from the origin and ending in the location with the said coordinates (Figure 1). Put this way, individuals are characterized by the directions and lengths of their person vectors. The personality space thus represents individual differences.

While techniques such as PCA attempt to identify popular directions among the person vectors, the PSF can use personality space for a wider range of purposes. First, personality space can be extended in "time" by developing through a (unlimited) number of cycles and person vectors can change their lengths and directions over this time (Figure 1). As a result, personality space does not only represent individual differences but also within-individual variation. Second, in addition to individuals themselves, every influence acting on each individual, situational or otherwise, can be represented as a vector in this space. We can call these force vectors. After all, for any change to happen there has to be some force(s) driving it. We will show that force vectors of whatever number and nature can continuously combine into individual-specific networks of connections among personality traits and thereby change the traits.

Under the model, force vectors influence person vectors by pressuring them to move in particular directions as the personality space develops through time. For example, the force vectors can represent both internal (e.g., genotypes) or external (e.g., observations of other individuals in the space or whatever situational perceptions) influences and they can impact individuals both additively and in transactions with each other. The force vectors can be timeinvariant (e.g., representing one's DNA sequence) or time-varying, and they can pertain to (i.e,

1 Naturally, the framework can be extended to describe situations where the sets of extant, or at least focal, traits differ across people or over time: if a trait is not relevant to an individual at a particular time, it can be 'silent' by having a zero value. 
have non-zero values for) all traits or only a subset of them (i.e., have values of zero for the nonpertinent traits). Likewise, individuals themselves can influence the forces acting on them, allowing for person-environment transactions (Caspi \& Roberts, 2001). This may sound like a sweeping and vague proposition (the kind that verbal theories often make), but we can operationalize this: individuals can influence other people around them by becoming force vectors for them, whereas these other people can be force vectors for them. This very simple principle means that people create their own social environments that then reinforce the characteristics (because people contributed these to the social environment). This, of course, is nothing else than an specific operationalization of the corresponsive principle.

It may look like the personality space can model a very complicated system—and indeed it does. Everything can influence everything else. Should we be overwhelmed by this possibility of a nearly infinite number of possible personality features transacting with a nearly infinite number of forces in a nearly infinite number of ways and thereby drop the idea as useless for any sensible purpose? We do not think so. It may not be useful to focus on specific personality features - their nature, number, or whatever property - or particular forces that may transact with other particular forces. But it may be very useful to think of possible organizational principles that could govern the dynamics of the system as a whole. If we can conceive of and operationalize (which requires very rigorous and mechanistic thinking indeed) some of these general principles and this seems to move the system through states, or towards a state, that reflect some phenomena in the real world, then we may have learned something about how personality could work. Focusing on general principles makes it unnecessary to enumerate the infinite number of specific transactions going on in the system.

Specifically, we propose that the dynamic processes that take place within and between 
individuals can have an overarching purpose of striving for an equilibrium or attractor state. This state corresponds to a balance between all influences acting on any individual at any given time, appropriately force-weighted, and the individuals' person vectors corresponding to this balance (cf. Cramer et al., 2012). Specifically, an individual's equilibrium can be operationalized as the combined effect of whatever forces acting on that individual no longer causing any change in their personality. Given some stability in these forces (e.g., due to genetic make-up or at least some stable components of environment), individuals generally tend to move closer to the state of equilibrium, although they are open to perturbations that can, at any time, redefine what the equilibrium state would be. We believe this to be one possible operationalization of the popular concept of personality maturation, whereby individuals generally become more stable, functional and socially and emotionally adjusted over time (Caspi \& Roberts, 2001; Caspi et al., 2005). This may be because most (although certainly not necessarily all) individuals are increasingly efficient in adjusting to the different influences acting on them, be these internal or external. Importantly, if individuals transact, their individual strivings for personal equilibrium are inherently intertwined, which results in tendency for the whole personality space to tend towards an equilibrium.

In equilibrium, there would no longer be any change, but of course this is only an idealized state towards which the system generally tends to move. Humans and their groups are not closed systems - all sorts of things can and do happen. In reality, new forces (e.g., external influences such as new individuals or information, or internal factors such as hormonal changes) can arrive on the scene and redefine the equilibrium. As discussed above, there may also be random influences. But even if the personality space only strives towards this state without reaching it, this can generate systematic patterns in apparently very complex systems. Of course, 
there are many processes through which equilibria can emerge from transactions in personality space. But exploring this is exactly our intent in designing the models. We can compare the patterns of processes and equilibria we observe to developmental and structural patterns from empirical data: for example, increases or decreases in trait-level variances in populations over time.

\section{Connections among traits}

In the PSF, an important aspect of an individual's personality is how his/her traits are inter-connected and thereby influence each other. This defines the individual's underlying personality structure (which does not necessarily correspond to the factor structure of individual differences among individuals). The inter-connections can mathematically be represented by a matrix of connection weights. Over time, the connections determine the relative trait scores, because the connections represent the force vectors through which traits act on each other. Under this model, we can think of personality as an amount of psychological activity or resources, which the connections allocate across the traits.

In models that describe one or, at best, a few measurement occasions for each participant, associations between variables can be represented as linear. However, when modeling dynamic systems based on the very large numbers of hypothetical causal processes per participant, as is possible to generate using computer simulations and that may mimic what happens in real world, one immediately faces the need to posit non-linear processes. The elements of a dynamic system simply cannot continuously influence each other in an invariant way. For example, if traits contribute to one another monotonically (as we would represent the associations in a typical structural/measurement model), their scores will grow unboundedly. Likewise, if the connections 
are negative and traits inhibit each other, levels of the traits could quickly shrink to near-zero. These are unlikely scenarios for psychological processes, at least pertaining to normal development. To avoid this, traits have to have either natural boundaries, negative feedback loops or both positive and negative connections that balance each other. Of note is that in a simulation study of personality stability by Fraley and Roberts (2005) appeared to mechanically constrain the variances to avoid the problem.

Thinking of personality processes as allocation of psychological resources across traits may be a parsimonious solution to this problem. Provided that the level of these resources is relatively stable over time — which really is just another way of saying that personalities neither vanish nor explode - then allocating more activity to some traits may automatically mean that there is less of it for other traits. Positive connections in some part of the personality system have to be accompanied by negative connections in another part. The limited resources can be thought of as effort, time, attention/focus, invested learning/practice, self-regulation or the likes (cf. Penke, 2010) and their amount may vary across people.

We can now see two ways of thinking of individuals' personalities: as person vectors in personality space that represent variations across individuals and over time, and causally connected systems within individuals. Moreover, we can see the two representations of personality as inherently inter-connected. Connections within individuals' personality systems control their positions in personality space by increasing the scores of some traits at the expense of others and thereby determining their relative rankings - both within- and across individuals. This way, individuals' internal personality structures become manifest as personality variability over time and across people. 


\section{Forces Can Influence Connections}

As personality change happens because of traits increasing or decreasing each other's value, forces operating on an individual's personality have to exert their influences by contributing to the connection matrix of their traits. Such forces being represented as multiple (force) vectors, there must then be a mechanism that combines the vectors and translates them into matrices.

As already specified, each force vector represents a location in personality space that attracts or repels the person vector at the particular point of time. The forces can also vary in their relative power (weights); for example, the force weight representing genetic influences may be weighed by some sort of "heritability" estimate. At each point, the person vector attempts to move towards a location that balances all these forces as per their relative weights at this time, being their weighted resultant which basically is a weighted average. This balance can be called a target vector. Naturally, the target vector can change over time as a result of changes in force vectors or their weights (Figure 2). Importantly, this target vector can be mathematically interpreted as the principal eigenvector of the connection matrix. This is a key idea of the PSF.

Matrices can be decomposed into eigenvectors and corresponding eigenvalues; this is the basis for PCA, for example. By the same token, given one or more eigenvectors and their corresponding eigenvalues, a matrix can be 'reconstructed' from them with varying degrees of precision. Here, the target vector is the eigenvector from which the connection matrix is to be reconstructed. The precision of this reconstruction can be (although does not have to be) systematically controlled and it could be seen as a measure of personality maturation, so that the more mature a personality, the more aligned it becomes with its target vector. This way, the more 
mature a person is, the more effectively he/she has adjusted his/her personality to all the forces acting on him/her (including the forces that reflect the persons transactions with environment). Thus, the PSF can model the convergence of person vectors towards their individual and collective equilibria as a gradual process, reflecting possible inertia in personality change.

Crucially, there is two-way traffic between person vectors and their related force vectors in personality space. Different force vectors combine into a target vector, which becomes the basis for the connection matrix, which in turn influences the person vector. What is more, the person vector can become a force vectors for other individuals' person vectors. [Naturally, the person vectors can influence whichever other force vectors researchers want to specify, too (e.g., an individual may choose a job whose characteristics match his/her personality traits, so a force vector reflecting job demands can become influenced by person vector), but we are not modeling these possibilities here.] This means that a person may change, or become part of, his/her own environment, which then influences him/her. These relatively simple rules allow for considerable flexibility in building specific personality models because the forces can continuously change, interact and be weighed differently as per researchers' hypotheses - but do this in a principled way and by producing observable parameters such as magnitudes of individual differences.

\section{Mathematical Specification of the PSF}

A person vector is represented numerically by a vector $\mathbf{y}$ of $k$ elements, which can be thought of as a multivariate score representing the observed personality characteristics represented by the $k$ traits, and a $k$ x matrix denoted $\mathbf{P}$ in which each element $a_{i, j}$ is a connection weight representing the amount of influence of the $i$ 'th trait upon the $j$ 'th trait, (or of the $i$ 'th trait upon itself for elements $a_{i, i}$. Individuals change their location $\mathbf{y}$ in personality space when their 
internal structure $\mathbf{P}$ changes. This is because $\mathbf{P}$ is not only a connection weight matrix but also a projection operator-it projects, or pulls, $\mathbf{y}$ towards a certain direction and length (the target vector). Matrix $\mathbf{P}$ is not necessarily symmetric and the connection weights may be positive, negative or zero. The rows of $\mathbf{P}$ represent contributions of the respective traits to other traits, whereas the columns encode the contributions that the respective traits receive. The diagonal values of $\mathbf{P}$ represent the stability of the corresponding traits. Because $\mathbf{y}$ represents the coordinates of an individual's location in personality space, we can think of personality change, individual differences (e.g., topological "distance" between personalities) and their clustering as projections across the space.

$\mathbf{P}$ acts as a projection operator by projecting the person vector $\mathbf{y}$ onto a target vector $\mathbf{v}$, the weighted resultant of the set of vectors representing every force acting on $\mathbf{y}$. As discussed above, the person vectors of one or more individuals may be combined into the target vector $\mathbf{v}$ alongside potentially unlimited number of other forces represented as vectors of length $k$. The matrix defined by $\mathbf{P}=\mathbf{A}\left(\mathbf{B}^{\mathrm{T}} \mathbf{A}\right)^{-1}$. $\mathbf{B}^{\mathrm{T}}$ is an oblique projection operator such that $\mathbf{P}$ projects $\mathbf{y}$ along a path specified by the orthogonal complement of the subspace spanned by $\mathbf{B}$ onto a target that is the subspace spanned by the columns of $\mathbf{A}$. Specifically, to construct an operator matrix $\mathbf{P}$ to project $\mathbf{y}$ onto a single spanning line defined by a target direction $\mathbf{v}$ ('rank-1' projection), matrix A needs only one column, which is $\mathbf{v}$. Matrix $\mathbf{B}$, the null space of $\mathbf{A}$, is used to define the path of the projection. The most parsimonious way of defining $\mathbf{B}$ is based on the rank-1 oblique projection along a straight line. In this case, the person vector $\mathbf{y}$ tends towards the direction and length of its target vector $\mathbf{v}$ along the minimum distance path (Figure 3). In order to obtain this, matrix $\mathbf{B}$ is specified as the set of column vectors that are orthogonal to $\operatorname{span}\{\mathbf{v}-\mathbf{y}\}$, which can be derived by a 
Gram-Schmidt process.

For computational reasons, the time-varying dynamics of personality space is divided into discrete steps (cycles); their "duration" is completely flexible. An individual's trait scores $\mathbf{y}_{n}$ and their connections $\mathbf{P}_{n}$ are time-varying, where $n$ denotes the time-point. The evolution of trait scores is related to the connections among them by a simple iteration scheme, $\mathbf{y}_{n}=\mathbf{y}_{n-1} \mathbf{P}_{n-l}$. According to this scheme, at each time-point, $n=1,2 \ldots$, the score of each trait is updated as the sum of all trait scores in vector $\mathbf{y}$ weighted by their respective influences upon the trait. This ensures that $\mathbf{y}_{n}$ is projected towards $\mathbf{v}_{n}$, as $\mathbf{P}_{n}$ is defined by $\mathbf{v}_{n}$. If matrix $\mathbf{P}$ were constant over time, it would be possible to condense the iteration to a single step involving the matrix power $\mathbf{P}^{n}$. The purpose of iterating with multiple steps, however, is to allow $\mathbf{P}_{n}$ to be time-varying and thereby to change as a result of dynamics in the personality space. The person vector $\mathbf{y}$ no longer changes when the weight matrix $\mathbf{P}$ is idempotent (i.e., $\mathbf{P}^{n}=\mathbf{P}^{n+l}$ ). To allow projection of $\mathbf{y}$ towards $\mathbf{v}$ to be gradual and thereby $\mathbf{v}$ to change along the way, however, it is necessary that $\mathbf{P}_{n}$ gradually approach idempotence. This property can be achieved as follows.

If matrix $\mathbf{P}$ can be decomposed into the eigenvectors collected into the matrix $\mathbf{Q}$ and corresponding eigenvalues encoded in the diagonal matrix $\boldsymbol{\Lambda}$, such that $\mathbf{P}=\mathbf{Q} \mathbf{\Lambda} \mathbf{Q}^{-1}$ (as is done in PCA), then $\mathbf{P}^{n}=\mathbf{Q} \mathbf{\Lambda}^{n} \mathbf{Q}^{-1}$ (cf. Meyer, 2000). This shows that as $n$ increases $\mathbf{P}^{n}$ keeps the same eigenvectors as $\mathbf{P}$, but the eigenvalues of $\mathbf{P}^{n}$ are $\boldsymbol{\Lambda}^{n} ; \mathbf{P}^{n}$ becomes idempotent when $\boldsymbol{\Lambda}^{n}$ contains eigenvalues of only 0 and 1 . Therefore, $\mathbf{P}$ can be made to approach idempotence by setting the toapproach-zero eigenvalues of $\mathbf{P}^{n}$ to fractions inside the unit circle and ascribing the to-remainnon-zero eigenvalues 1 . Then, as $n$ increases, the eigenvalues of $\mathbf{P}^{n}$ that are 1 stay constant, but the fractional eigenvalues tend to 0 , so that $\mathbf{P}^{n}$ becomes idempotent in the limit. The rate of 
convergence to idempotence is controlled by the size of the fraction: if the fractional eigenvalues are already 0 , then convergence is immediate, whereas the closer they are to -1 or 1 , the slower the convergence, because larger $n$ is needed to reduce them. Armed with this, we can see that if one of the columns of $\mathbf{Q}$ is $\mathbf{v}$ (the other columns can contain random numbers) and only the corresponding eigenvalue in $\Lambda$ is 1 (the other eigenvalues fractional within the unit circle), the iteration causes $\mathbf{y}$ to approach $\mathbf{P}^{n} \mathbf{y}$ (i.e., $\mathbf{v}$ ) in ever decreasing steps (Figure 3).

\section{Employing PSF to gauge changes in trait variance}

PSF provided us with a computational framework for exploring how situational influences in the form of a) person-environment transactions and b) random influences can, in principle, play out in terms of variability in personality characteristics, all other things kept constant.

We first explored the possible impact of person-environment transactions. Here, environment means accumulating situational experiences resulting from other people. Specifically, this referred to individuals (whom we call agents) interacting with the most similar other agents (whom we call friends) in the personality space. Of course, in real life people do not only interact with people why prefer to, but we modeled the idea that generally people are more likely to gravitate towards or end up in similar situations with like-minded others; perhaps the non-preferred others can be considered random influences that we will also address. The similarity between agents was operationalized as the absolute distance across all traits (sum of squared differences between respective scores). The interactions were operationalized as agents incorporating the trait levels of their friends among their own force vectors, amounting to friends personalities influencing their personality. Importantly, their friends would tend do the same because the same principle applied to all agents and the highest similarity tended to be mutual. 
This meant that in addition to being directly influenced by their self-selected social environment, agents' own traits automatically became part of this social environment (because their traits influenced those of their their friends). This simple principle meant that agents not only selected but also carved themselves (social) environments that matched and reinforced their pre-existing traits. These interactions happened iteratively (computationally, through cycles) and could be thought of as mimicking rather mundane, everyday-like situational influences. The processes were not deterministic in the sense that at every step agents' scores could change and thereby the equilibrium towards which each agent (and the whole personality space) strived could also change. We also allowed a small amount of random influences (small shocks) at every step, so that the personality space could never reach a complete equilibrium-agents would only wiggle themselves more of less close to the locations in space where they felt comfortable in. We varied the number of friends people interacted with. We then explored the impact of random influences, in which every agent mostly received influences unrelated to their own and others' pre-existing traits.

The setup

In all simulations, 1,000 agents were operationalized as vectors in a 50-dimensional personality trait space; that is, each agent was characterized by 50 traits. This is of course an arbitrarily small number of traits, but we had to settle on a number and substantially increasing it would have entailed computational costs. Each trait was normally distributed with a zero mean and unit variance. The development of personality space was modeled through 100 cycles, which appeared sufficient for stable patterns to emerge.

At each cycle, each individual was subject to three forces. First, there was an invariant 
force vector with a constant weight of 0.5 , which represented a constant pull towards a certain baseline (cf. DeYoung, 2015). This constant baseline towards which agents persistently gravitated could be thought of as reflecting, among other things, genetic or stable pre- and perinatal influences, or other influences stemming from early and stable aspects of environment. Reducing the weight of this constant force or ditching it altogether would have resulted in other processes playing out more strongly. The second force vector represented the combined influence of the agents' friends at the particular cycle, operationalized as their average trait levels at this point. We varied the number of friends $(5,10,100$ and 500). The third force vector represented random influences that were redefined at each cycle for each individual and operationalized as a vector of random numbers drawn from a normal distribution with zero mean and unit variance. The weights of the latter two vectors were subject to manipulation (either 0.40 and 0.10 for the person-environment transaction condition or 0.10 and 0.40 for the random influences condition, respectively). The convergence rate towards the equilibrium (eigenvalues other than the one associated with the principal eigenvector) was set at 0.90 , representing relatively slow convergence. This left enough wiggling room in the personality space for the transactions to play out over time.

Due to relatively high computational demands of the models, we only ran each simulation once. All scenarios were based on exactly the same initial values (agents' scores), so crosssimulation differences could not reflect differences in the start values. The simulations were carried out in R statistical language (R Core Team, 2016) and the scripts are made publicly available in Open Science Framework repository (osf.io/pzat9). This means that anyone with a basic understanding of the R statistical language can explore and modify the models. 
The results

As for person-environment transactions, the results of the simulations contradicted the hypothesis put forward previously (Mõttus et al., 2016), whereby the transaction (as per corresponsive principle) would lead to an increase in the magnitude of individual differences. According to these simulations, the transactions appeared to decrease the variance of agents' characteristics (Figure 4). The average standard deviation across 50 characteristic was initially 1 (as per model setup), but it quickly dropped to around .60 and then somewhat recovered to between .70 and .80 . Moreover, there appeared a systematic, albeit weak, trend for interactions with a greater number of other agents conferring a greater decrease in the magnitude of individual differences.

With the benefit of hindsight, we can look for a possible explanation for such a pattern of findings. After all, this is exactly what computational models are for. One has an idea, one runs a proof-of-principle test of it by implementing it as a computational model, one finds out that the idea may not be as plausible as initially thought — and one goes back to the drawing board to come up with a new idea. For example, it is possible that the observed effect of decreasing variance as a result of social interactions can be ascribed to regression to the mean. Specifically, even if individuals select and carve out matching social niches that reinforce their pre-existing traits, it is likely that extreme pre-existing trait levels are not going to be matched with equally or even more extreme levels, simply because extreme levels are rare-most individuals have rather moderate trait levels. That is, if traits, and thereby resulting social experiences of those that observe these traits, are normally distributed then moderate experiences tend to be statistically more likely than extreme experiences. 
This explanation is consistent with the observation that interacting with a greater number of friends contributed to differences between agents becoming even smaller than interacting with fewer others. This is because the more others an agent observed, the more likely it was that the average trait levels of these others veered towards the population mean-even if the others were selected based on the agent's own traits, however extreme these were. For an agent with extreme trait levels, amassing a group of friends who were equally extreme as the agent itself was only possible when the intended group of friends was small — again, because there simply were not enough agents with extreme trait levels to interact with. The bigger the group of friends, the more of an "average" agent they tended to be, on average.

As for the scenario where the time-varying situational influences were random, the results were consistent with the prediction (Mõttus et al., 2016): these influences tended to confer a decrease in the magnitude of individual differences such that an initial sharp drop was followed by a partial recovery (Figure 5). The changes in variance resulting from random influences were almost identical to those resulting from interactions with a large number of of friends. This is probably because random influences were distributed normally (which they also might be in real life) and the trait level distributions of a large group of friends were also likely to be normally distributed with means not far off from population means, as was discussed in the previous paragraph.

Interestingly, in each scenario we observed a somewhat deeper decrease in the magnitude of individual differences at the beginning of agent's "life-cycles" followed by a gradual albeit partly recovery of this property. This is reflects two things. First, it resulted from agents being more open to influences at earlier cycles because of being relatively further away from their 
target vectors which to a substantial extent represented their time-invariant baseline trait levels. As a result, the multiple influences that collectively tended to pull agents towards population mean scores could have a stronger effect at earlier cycles. By later cycles, agents would have wiggled themselves increasingly closer to their niches in personality space (partly reflecting the time-invariant baseline) and they also tended to be less open to variance-decreasing influences. Second, the initial values of agents' scores were random in this setup. Had the initial values been identical to those of the time-invariant force vector (e.g., already represented what would be stable about the agents throughout) such an initial dip in the magnitude of individual differences would not have emerged, because agents would already have converged towards their would-be baseline values.

Of note is that the observed pattern is not inconsistent with empirical findings showing tendency for increasing magnitude of individual differences in childhood (Mõttus et al., in revision). So, among other things, the empirical findings may suggest that children do not start their personality development with what would be their more or less stable baseline trait levels but gradually gravitate towards these, becoming increasingly differentiated from their peers along the way. Of course, we emphasize that this is only a hypothesis. But it is a hypothesis that would perhaps not have occurred without such modeling.

The bottom line from these simulations seems to be: all else equal, the accumulation of a large number of situational experiences could constrain the variance of psychological characteristics, regardless of whether the experiences are systematically tied to pre-existing levels of the characteristics. Possibly, as we navigate through life and experience all sorts of situations, this may make us all a little more alike. 
A corollary finding: The crud factor

Such simulations may incidentally lead to observations that were not initially planned, such as the initially even more accentuated dip in variance described above. These corollary observations might be useful for generating novel hypotheses. For example, we also noticed that the accumulation of person-environment transactions contributed to the correlations among the characteristics. Figure 6 plots the first eigenvalues of the correlation matrices among the 50 characteristics. These eigenvalues increased in when social interactions were the dominant timevarying influence on characteristics but barely increased when social interactions were less prominent and random influences dominated. This suggests that individuals' characteristics may became more inter-correlated as a result of person-environment transactions.

It is in fact easy to see why social interactions could contribute to correlations among characteristics. There being a pervasive pattern of correlations among characteristics (in other words, a principal component) means that one direction of person vectors is more popular than other directions in the personality space, as was discussed above. When agents interacted, they gravitated towards each other and inevitably some directions became more popular than others. To the extent that the interacting groups of agents partly overlapped, they were likely to eventually veer towards a somewhat common direction-a what would become a normative (personality) profile for this population.

This is what happened in the simulations but the scenario may also have a real-world counterpart. Specifically, it has been noticed for a long time (e.g., Thorndike, 1911) that there is a pervasive pattern of correlations among the variables that psychologists (and social scientists more generally) measure. Among other things, this has been called the crud factor (Meehl, 1990) 
or ambient noise level (Lykken, 1968). "Everything correlates to some extent with everything else" (Meehl, 1990, p. 204), and it does not necessarily result from methodological artefects but may reflect "real differences, real correlations, real trends and patterns for which there is, of course, some true but complicated multivariate causal theory" (Meehl, 1990, p. 208). The current simulations suggest that the accumulation of systematic (social situational) experiences may be one of the mechanisms that contributes to the crud factor.

\section{Did we need PSF for all this?}

Could such analyses have been carried out in a simpler way? Maybe. For example, in order to explore the effect of random (situational) influences on the variance of personality characteristics we could have carried out the simplest possible simulation according to which a trait $(\mathbf{y})$ is iteratively updated by a random value: $\mathbf{y}_{t}=\mathbf{y}_{t-1}+\mathbf{r}$, where $t$ the denotes time and $\mathbf{r}$ a random variable (for example, $\mathbf{r}$ could have the the same distribution as $\mathbf{y}$ ). This is essentially a random walk model. However, implementing such a simple model in any computational device, one will immediately see that it does not work. This is because the variance of $\mathbf{y}$ will monotonically increase. For example, with $t=10$ the standard deviation of $\mathbf{y}$ may have increased ten-fold and with $t=1000$ it may have increased thirty-fold. This is not realistic. Hence, such a simplistic linear model that never converges is unlikely to be a good representation of personality processes. Of course, one may deliberately constrain the variance by rescaling the variables at every cycle (cf. Fralye \& Roberts, 2005), but this would automatically undermine any attempt too investigate changes in variance. A framework such as PSF that accommodated non-linear dynamics and self-organizational processes may be more suitable for building such models. 


\section{Conclusions}

It has been previously hypothesized that, ceteris paribus, systematic person-environment transactions should entail increases in the magnitude of individual differences (Mõttus et al., 2016). That is, when people choose which situations to attend and how to shape and interpret these situations, they are likely to do this in ways that match their pre-existing characteristics, and these self-selected/created experiences are then likely to accentuate the pre-existing characteristics even more. As Roberts and colleagues (2003) put it: "the most likely effect of life [situational] experience on personality development is to deepen the characteristics that lead people to those experiences [situations] in the first place." However, although there is evidence for increases in the variance of personality characteristics until early adolescence, the magnitude of individual differences appears relatively constant thereafter. Among other possible explanations, it has been hypothesized that this may be because an allegedly variance-decreasing effect of random influences countervails the allegedly variance-increasing effect of personenvironment transactions (Mõttus et al., 2016).

The current simulations, however, suggest that the intuition might have been wrong. Perhaps the accumulation of systematic situational experiences does not entail the accentuation of individual differences in the sense of increasing variance after all. And yet the accumulation of such experiences may still influence individuals characteristics in systematic ways as was suggested by increases of the correlations among agents' characteristics. This may suggests that individuals do carve themselves social niches that match their pre-existing characteristics and thereby deepen these characteristics, but such accentuation of individual differences reflects more subtle repositioning in the personality feature space rather than linearly growing in particular 
directions. Thus, the simulation results may in fact reconcile the corresponsive principle and current empirical findings pertaining to developmental trends in the magnitude of individual differences. Of course, the question remains as to the reasons for the increases in personality variance in children. Among other thing, this may reflect gradually converging towards what would be the baseline trait levels thereafter, as appeared in our simulations. Alternatively, this may reflect the enrichment of children's behavioral repertoires, for example.

In conclusion, we hope that the chapter provided support for two ideas. First, we hope that our example showed how computational modeling generally allows playing through different scenarios and how this may help us in thinking about complicated phenomena. Of course, we have to emphasize again that no one should think of computational models as providing evidence for an idea. They can only guide thinking, motivate rethinking, point us to new directions and perhaps help us to estimate the relative plausibility of some ideas over others - in principle. Second, we hope that our examples showed how computational modeling can be specifically used to think about the complex interplay between individuals and their situational experiences. It is extremely likely that individuals and the situations that they experience transact in pervasive and yet complicated ways. Furthermore, the transactions are likely to play out across a large number of more or less discrete encounters and be non-linear in that a situational feature does not always influence a trait in a constant way or the other way around - traits do not always influence situations in the same way. Computational modeling may be well suited for operationalizing this complexity in rigorous ways, pitting different scenarios against each other and perhaps coming up with novel ideas. 


\section{References}

Asendorpf, J. B. (2015). Person-centered approaches to personality. In M. Mikulincer, P. R. Shaver, M. L. Cooper, \& R. J. Larsen (Eds.), APA handbook of personality and social psychology, Volume 4: Personality processes and individual differences (pp. 403-424). Washington, DC, US: American Psychological Association.

Bahns, A. J., Crandall, C. S., Gillath, O., \& Preacher, K. J. (2016). Similarity in Relationships as Niche Construction: Choice, Stability, and Influence Within Dyads in a Free Choice Environment. Journal of Personality and Social Psychology. https://doi.org/10.1037/pspp0000088

Bjørnebekk, A., Fjell, A. M., Walhovd, K. B., Grydeland, H., Torgersen, S., \& Westlye, L. T. (2013). Neuronal correlates of the five factor model (FFM) of human personality: Multimodal imaging in a large healthy sample. NeuroImage, 65, 194-208. https://doi.org/10.1016/j.neuroimage.2012.10.009

Caspi, A., \& Roberts, B. W. (2001). Personality Development across the Life Course: The Argument for Change and Continuity. Psychological Inquiry, 12(2), 49-66. https://doi.org/10.2307/1449487

Caspi, A., Roberts, B. W., \& Shiner, R. L. (2005). Personality Development: Stability and Change. Annual Review of Psychology, 56, 453-484. https://doi.org/10.1146/annurev.psych.55.090902.141913

Cramer, A. O. J., van der Sluis, S., Noordhof, A., Wichers, M., Geschwind, N., Aggen, S. H., ... Borsboom, D. (2012). Dimensions of Normal Personality as Networks in Search of Equilibrium: You Can't Like Parties if You Don't Like People. European Journal of Personality, 26(4), 414-431. https://doi.org/10.1002/per.1866

DeYoung, C. G. (2015). Cybernetic Big Five Theory. Journal of Research in Personality, 56, $33-$ 58. https://doi.org/10.1016/j.jrp.2014.07.004 
Fraley, R. C., \& Roberts, B. W. (2005). Patterns of continuity: a dynamic model for conceptualizing the stability of individual differences in psychological constructs across the life course. Psychological Review, 112(1), 60-74. https://doi.org/10.1037/0033295X.112.1.60

Gershman, S. J., Horvitz, E. J., \& Tenenbaum, J. B. (2015). Computational rationality: A converging paradigm for intelligence in brains, minds, and machines. Science, 349(6245), 273-278. https://doi.org/10.1126/science.aac6076

Grosz, M. P., Dufner, M., Back, M. D., \& Denissen, J. J. A. (2015). Who is open to a narcissistic romantic partner? The roles of sensation seeking, trait anxiety, and similarity. Journal of Research in Personality, 58, 84-95. https://doi.org/10.1016/j.jrp.2015.05.007

Lykken, D. T. (1968). Statistical significance of psychological research. Psychological Bulletin, 70, 151-159. doi:10.1037/h0026141

Meehl, P. E. (1990). Why summaries of research on psychological theories are often uninterpretable. Psychological Reports, 66, 195-244. doi:10.2466/PR0.66.1.195-244

Meyer, C. D. (Ed.). (2000). Matrix Analysis and Applied Linear Algebra. Philadelphia, PA, USA: Society for Industrial and Applied Mathematics.

Mõttus, R., \& Allerhand, M. (in press). Why do traits come together? The underlying trait and network approaches. In V. Zeigler-Hill \& T. K. Shackelford (Eds), SAGE handbook of personality and individual differences: Volume 1. The science of personality and individual differences (pp. $\mathrm{xx}-\mathrm{xx}$ ). London: SAGE.

Mõttus, R., Allik, J., Hřebíčková, M., Kööts-Ausmees, L., \& Realo, A. (2016). Age Differences in the Variance of Personality Characteristics. European Journal of Personality, 30, 4-11. https://doi.org/10.1002/per.2036

Mõttus, R., Epskamp, S., \& Francis, A. (in press). Within- and between individual variability of 
personality characteristics and physical exercise. Jorunal of Research in Personality.

Mõttus, R., Soto, C. J., \& Slobodskaya, H. R. (in revision). Are all kids alike? The magnitude of individual differences in personality characteristics tends to increase from early childhood to early adolescence. Journal of Personality and Social Psychology.

Penke, L. (2010). Bridging the Gap Between Modern Evolutionary Psychology and the Study of Individual Differences. In D. M. Buss \& P. H. Hawley (Eds.), The Evolution of Personality and Individual Differences (pp. 243-279). Oxford University Press.

R Core Team (2016). R: A language and environment for statistical computing. R Foundation for Statistical Computing, Vienna, Austria. URL https://www.R-project.org/

Read, S. J., Monroe, B. M., Brownstein, A. L., Yang, Y., Chopra, G., \& Miller, L. C. (2010). A neural network model of the structure and dynamics of human personality. Psychological Review, 117(1), 61-92. https://doi.org/10.1037/a0018131

Roberts, B. W., Kuncel, N. R., Shiner, R., Caspi, A., \& Goldberg, L. R. (2007). The power of personality: The comparative validity of personality traits, socioeconomic status, and cognitive ability for predicting important life outcomes. Perspectives on Psychological Science, 2(4), 313-345. https://doi.org/10.1111/j.1745-6916.2007.00047.x

Selfhout, M., Burk, W., Branje, S., Denissen, J., van Aken, M., \& Meeus, W. (2010). Emerging late adolescent friendship networks and Big Five personality traits: a social network approach. Journal of Personality, 78(2), 509-538. https://doi.org/10.1111/j.14676494.2010.00625.x

Sherman, R. A., Rauthmann, J. F., Brown, N. A., Serfass, D. G., \& Jones, A. B. (2015). The Independent Effects of Personality and Situations on Real-Time Expressions of Behavior and Emotion. Journal of Personality and Social Psychology, 109, 872-888. https://doi.org/10.1037/pspp0000036

Strang, G. (2009). Introduction to Linear Algebra, Fourth Edition (4 edition). Wellesley, Mass: 
Wellesley Cambridge Press.

Thorndike, E. L. (1911). Individuality. Boston: Houghton Mifflin Company.

Wood, D., Gardner, M. H., \& Harms, P. D. (2015). How functionalist and process approaches to behavior can explain trait covariation. Psychological Review, 122, 84-11. 


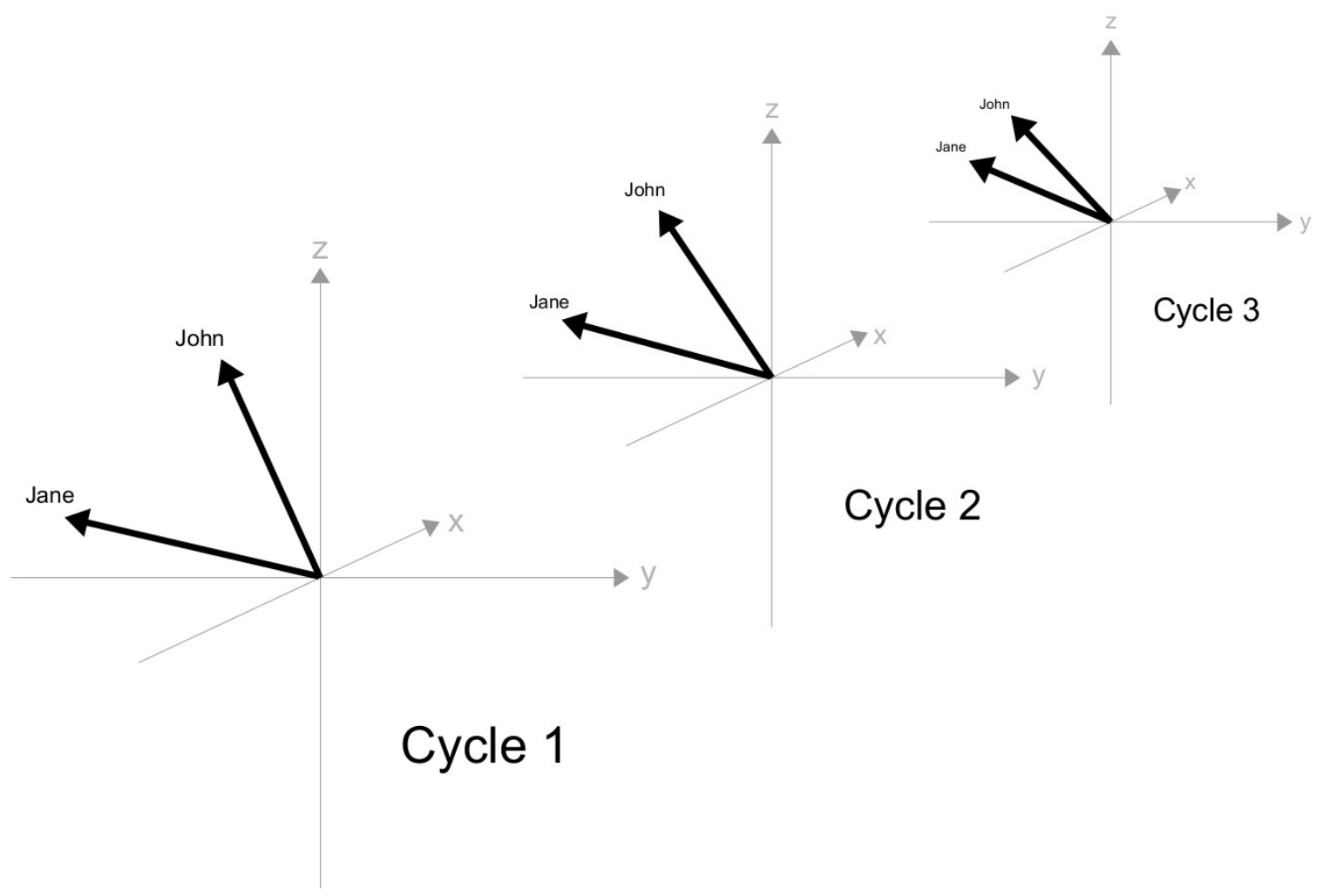

Figure 1. The development of personality space across three cycles. John and Jane become more similar over time. 


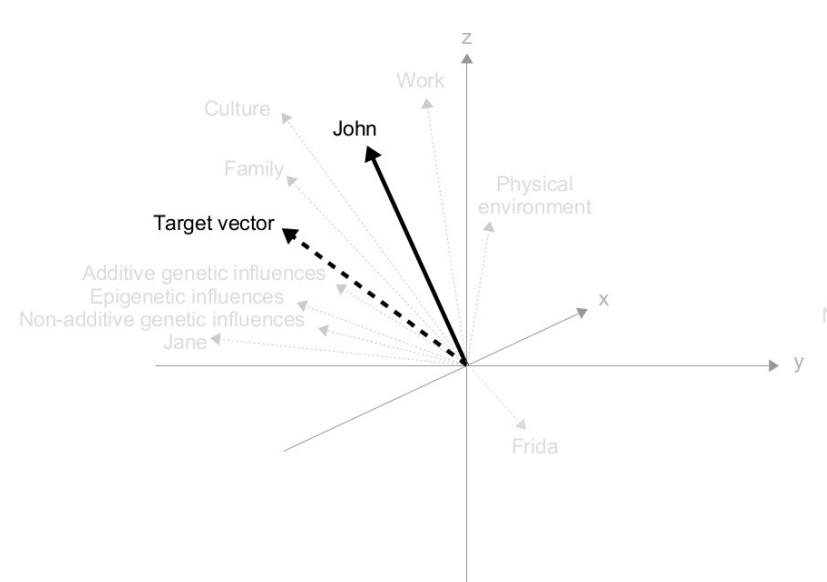

An earlier cycle

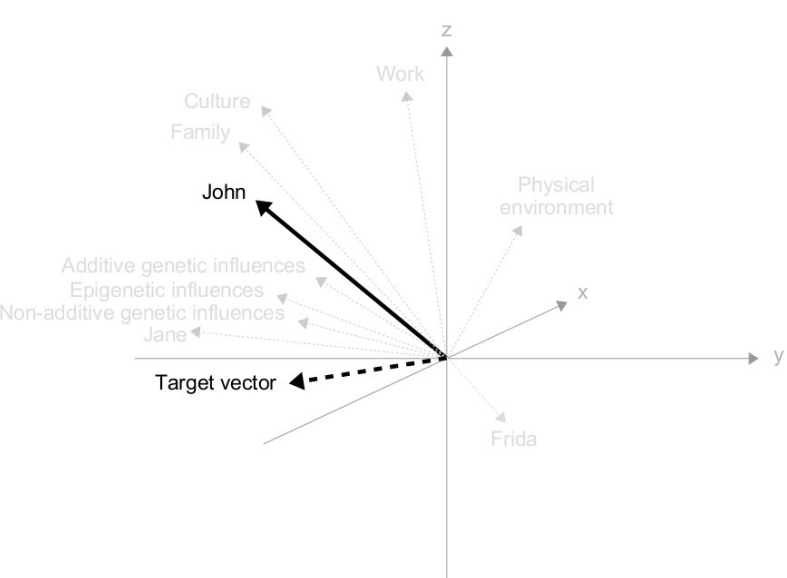

A later cycle

Figure 2. Multiple force vectors are combined into a target vector, which is their weighted resultant. The target vector changes as a result of changing forces or their weights. The figure conveys the idea that the number and nature of the force vectors that can combine into the target vector is unlimited, although in the chapter we really only consider three types of them. 


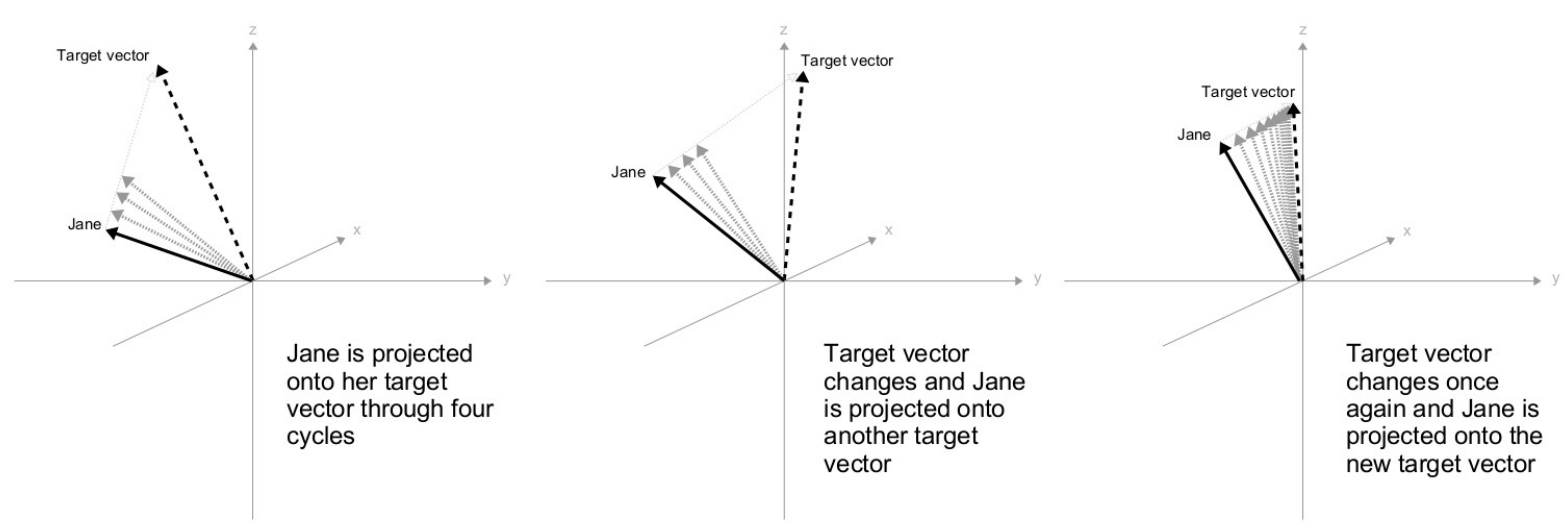

Figure 3. Convergence towards target vector is gradual. Jane is projected towards her target vector, which changes over time. As a result, the trajectory of the projection changes accordingly. 


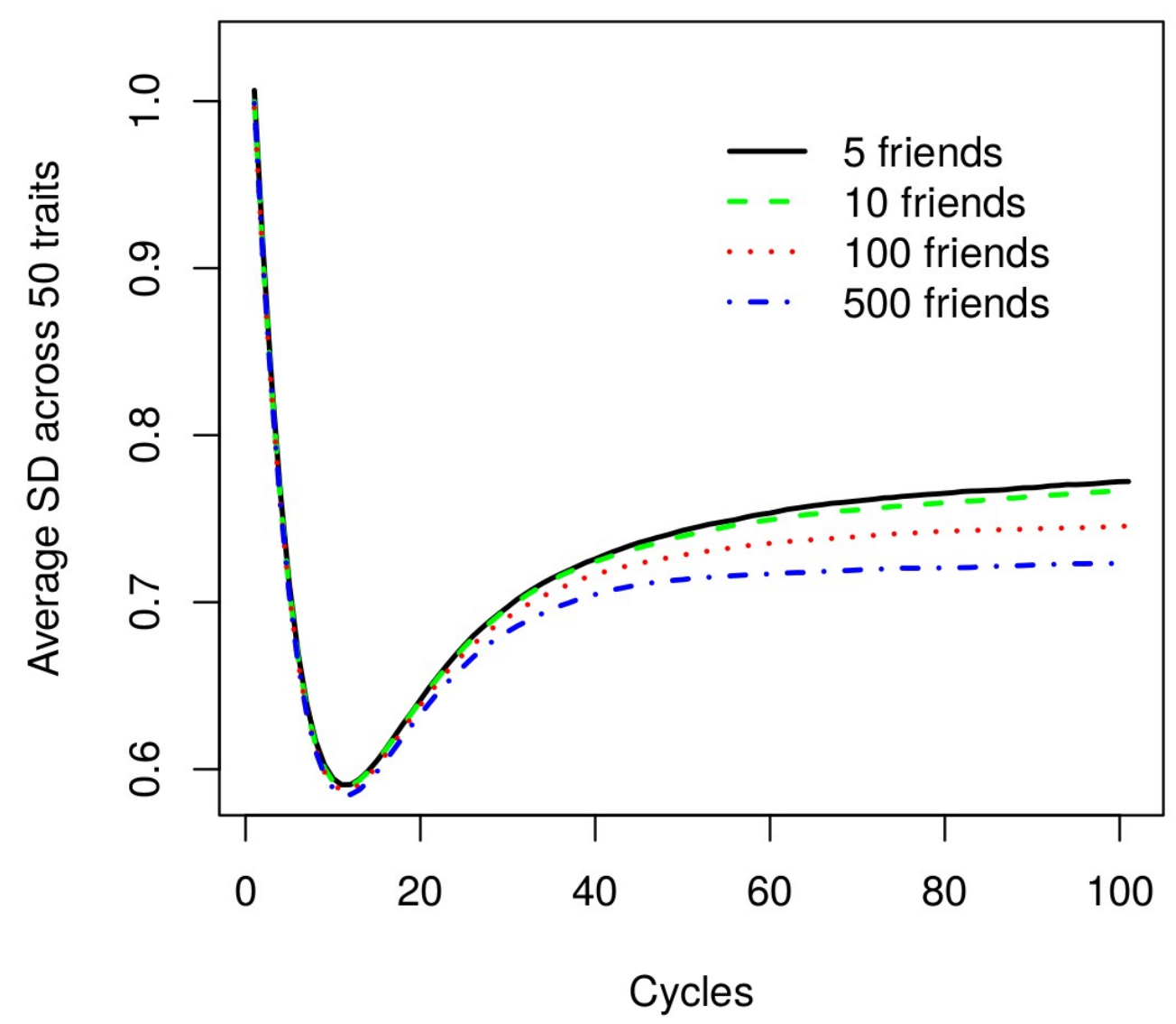

Figure 4. Average standard deviations of 50 personality characteristics across 100 cycles, when person-environment transactions involved different numbers of friends. 


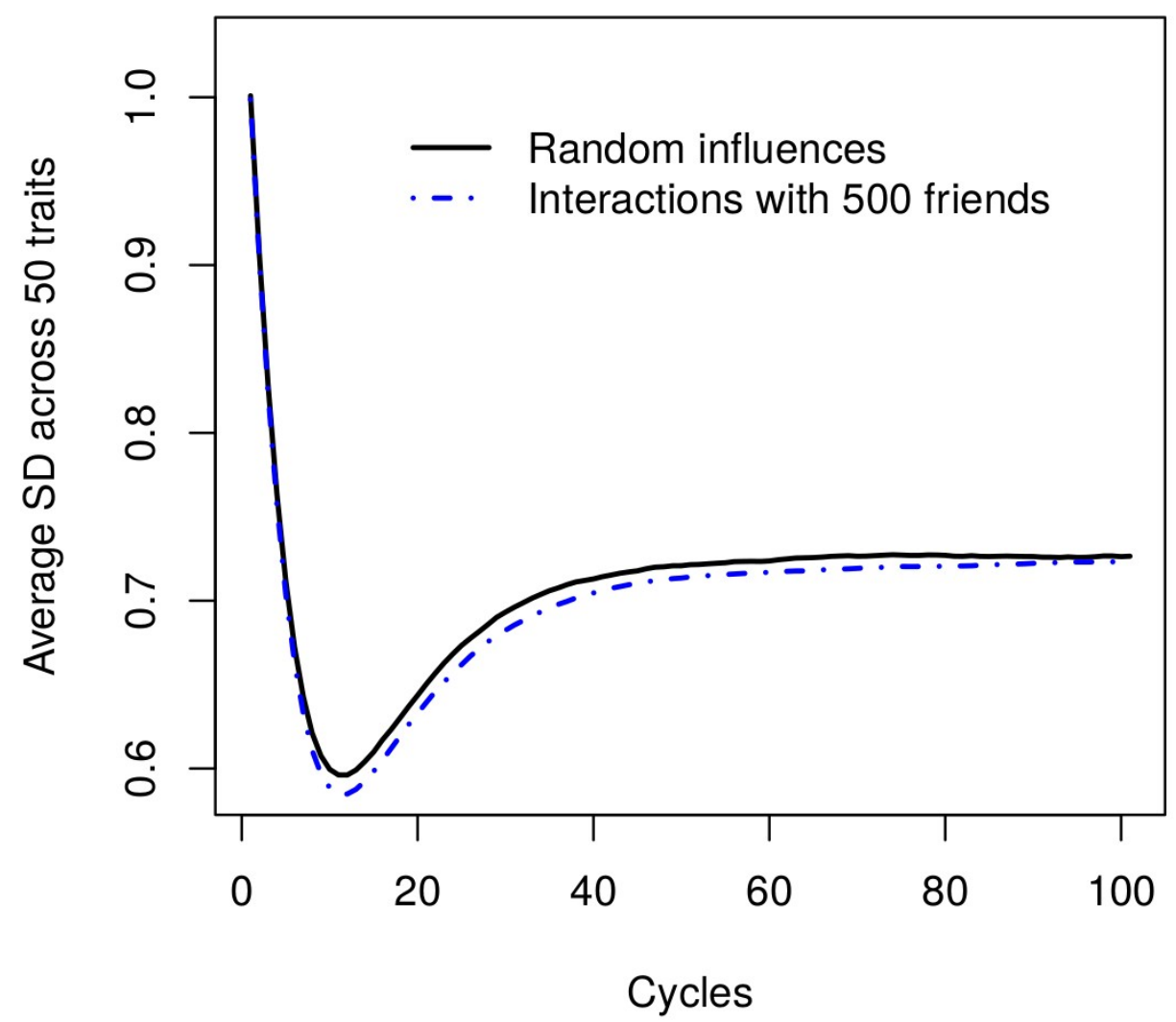

Figure 5. Average standard deviations of 50 personality characteristics across 100 cycles, when agents mostly experienced random influences. For reference, changes in standard deviations when person-environment transactions involved 500 friends are shown (same as in Figure 4). 


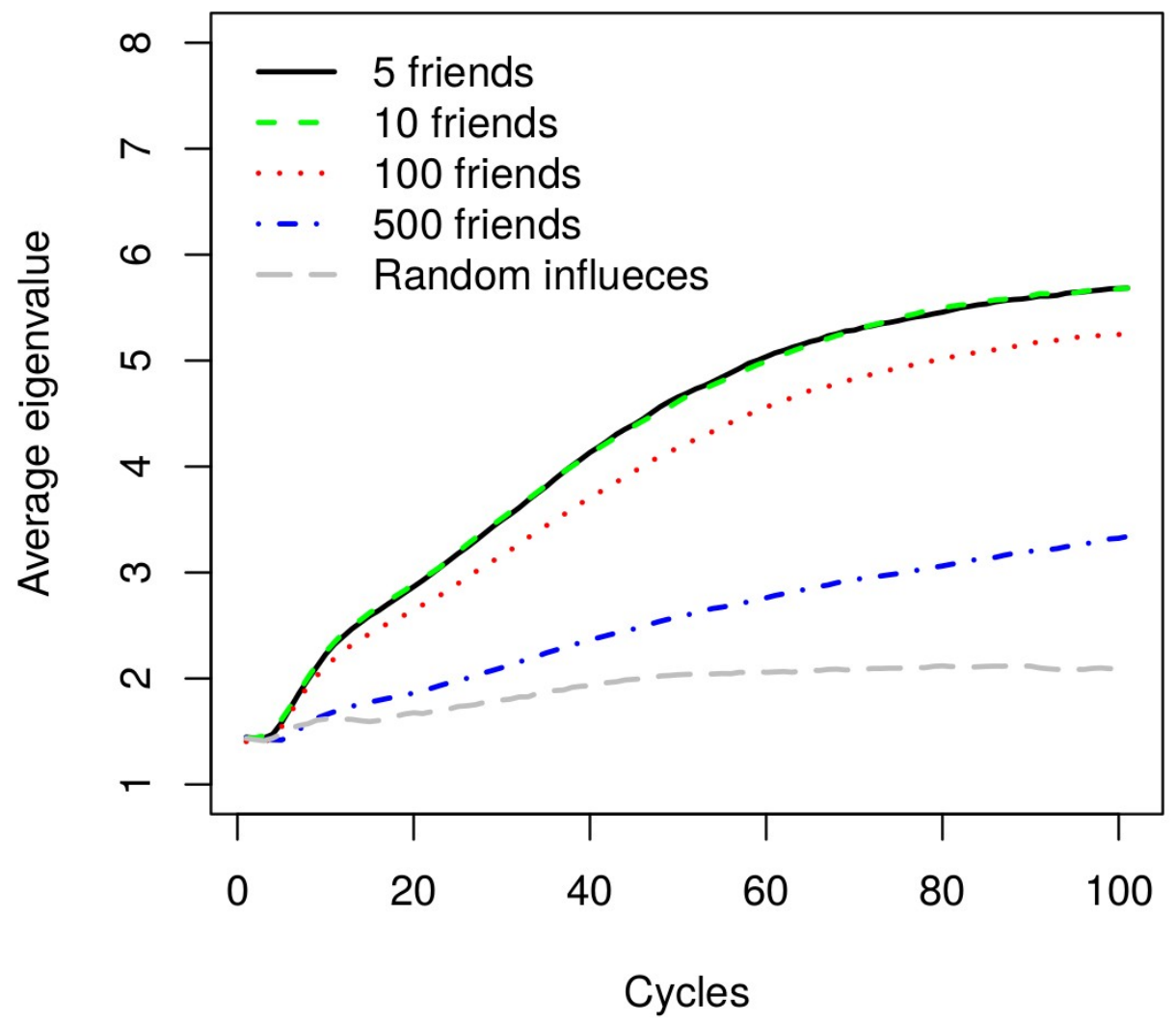

Figure 6. First eigenvalues of the correlations among 50 personality characteristics, when either person-environment transactions involved different numbers of friends or agents mostly experienced random influences. 